\title{
A multi-omics study links TNS3 and SEPT7 to long-term former smoking NSCLC survival
}

\author{
Sipeng Shen (iD) ${ }^{1,2,3,16}$, Yongyue Wei ${ }^{1,3,16}, \mathrm{Yi} \mathrm{Li}^{4}$, Weiwei Duan ${ }^{5}$, Xuesi Dong ${ }^{1,6}$, Lijuan Lin ${ }^{1,6}$, Dongfang You ${ }^{1,6}$, Adonina Tardon $^{7}{ }^{7}$,

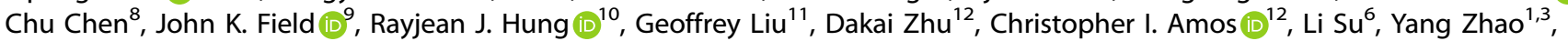 \\ Zhibin $\mathrm{Hu}^{2,3,13,14}$, Hongbing Shen ${ }^{3,13,14}$, Ruyang Zhang $\mathbb{D}^{1,3 凶}$, Feng Chen $\mathbb{D}^{1,2,3,14,17 凶}$ and David C. Christiani (iD) 6 ,
}

The genetic architecture of non-small cell lung cancer (NSCLC) is relevant to smoking status. However, the genetic contribution of long-term smoking cessation to the prognosis of NSCLC patients remains largely unknown. We conducted a genome-wide association study primarily on the prognosis of 1299 NSCLC patients of long-term former smokers from independent discovery $(n=566)$ and validation $(n=733)$ sets, and used in-silico function prediction and multi-omics analysis to identify single nucleotide polymorphisms (SNPs) on prognostics with NSCLC. We further detected SNPs with at least moderate association strength on survival within each group of never, short-term former, long-term former, and current smokers, and compared their genetic similarity at the SNP, gene, expression quantitative trait loci (eQTL), enhancer, and pathway levels. We identified two SNPs, rs34211819 $9_{T N S 3}$ at $7 \mathrm{p} 12.3\left(P=3.90 \times 10^{-9}\right)$ and $\mathrm{rs} 1143149_{\text {SEPT7 }}$ at $7 \mathrm{p} 14.2\left(P=9.75 \times 10^{-9}\right)$, were significantly associated with survival of NSCLC patients who were long-term former smokers. Both SNPs had significant interaction effects with years of smoking

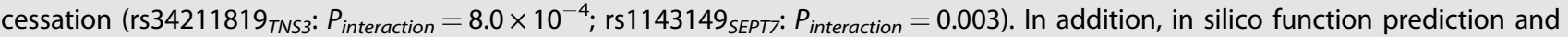
multi-omics analysis provided evidence that these QTLs were associated with survival. Moreover, comparison analysis found higher genetic similarity between long-term former smokers and never-smokers, compared to short-term former smokers or current smokers. Pathway enrichment analysis indicated a unique pattern among long-term former smokers that was related to immune pathways. This study provides important insights into the genetic architecture associated with long-term former smoking NSCLC.

npj Precision Oncology (2021)5:39; https://doi.org/10.1038/s41698-021-00182-3

\section{INTRODUCTION}

Non-small cell lung cancer (NSCLC) accounts for more than $85 \%$ of lung cancer cases and is the most commonly diagnosed malignant disease ${ }^{1}$. Tobacco smoking is a well-known environmental exposure leading to lung cancer ${ }^{2,3}$ and has been found to be linked to the majority of NSCLC deaths ${ }^{4}$. Emerging evidence shows that NSCLC in smokers and never smokers are different and separate entities ${ }^{5-7}$

It is, however, less well known that former smokers who quitted smoking long time before (e.g., >10 years), termed long-term former smokers, may also develop lung cancer ${ }^{8}$, and NSCLC patients who are long-term former smokers still harbor a high mortality risk ${ }^{9}$. So far, few studies have focused on the impact of genetic architecture on the prognosis of such a special group of patients, whose molecular mechanism for cancer death still remains unclear.

Leveraging the well-established International Lung Cancer Consortium (ILCCO) and Harvard Lung Cancer Study (HLCS), we conducted a GWAS by focusing on NSCLC patients who were longterm former smokers and identified germline genetic variants associated with overall survival time. We detected a set of SNPs with at least moderate association signals with survival from this subgroup as well as from other smoking subgroups, namely, never smokers, short-term former smokers, and current smokers. Comparing these SNPs, we investigated the genetic similarity across these smoking subgroups at the SNP, gene, expression quantitative trait loci (eQTL), enhancer, and pathway levels.

\section{RESULTS}

\section{Genetic variants are associated with survival}

The genome control inflation factor $(\lambda)$ for the overall population under the additive model was estimated to be 1.079 (Supplementary Fig. 1), which was comparable to a previous genomewide survival study ${ }^{10}$, indicating that the confounding effect caused by population stratification was well controlled. Two SNPs (rs34211819 at chromosome 7p12.3, and rs1143149 at $7 p 14.2)$ reached genome-wide significance among NSCLC patients who were long-term former smokers (Fig. 1a).

\footnotetext{
${ }^{1}$ Department of Biostatistics, Center for Global Health, School of Public Health, Nanjing Medical University, Nanjing 211166 Jiangsu, China. ${ }^{2}$ State Key Laboratory of Reproductive Medicine, Nanjing Medical University, Nanjing 211166 Jiangsu, China. ${ }^{3}$ China International Cooperation Center of Environment and Human Health, Nanjing Medical University, Nanjing 211166 Jiangsu, China. ${ }^{4}$ Department of Biostatistics, University of Michigan, Ann Arbor, Ml 48109, USA. ${ }^{5}$ Department of Bioinformatics, School of Biomedical Engineering and Informatics, Nanjing Medical University, Nanjing 211166 Jiangsu, China. ${ }^{6}$ Department of Environmental Health, Harvard T.H. Chan School of Public Health, Harvard University, Boston, MA 02115, USA. ${ }^{7}$ University of Oviedo and CIBERESP, Faculty of Medicine, Oviedo 33003, Spain. ${ }^{8}$ Program in Epidemiology, Division of Public Health Sciences, Fred Hutchinson Cancer Research Center, Seattle, WA 98109, USA. ${ }^{9}$ Institute of Translational Medicine, University of Liverpool, Liverpool, UK. ${ }^{10}$ Prosserman Centre for Population Health Research, Lunenfeld-Tanenbaum Research Institute, Sinai Health System and University of Toronto, Toronto, ON M5T 3L9, Canada. ${ }^{11}$ Princess Margaret Cancer Centre, Toronto, ON M5G 2 C1, Canada. ${ }^{12}$ Department of Medicine, Epidemiology Section, Institute for Clinical and Translational Research, Baylor Medical College, Houston, TX 77030, USA. ${ }^{13}$ Department of Epidemiology, Center for Global Health, School of Public Health, Nanjing Medical University, Nanjing 211166 Jiangsu, China. ${ }^{14}$ Jiangsu Key Lab of Cancer Biomarkers, Prevention and Treatment, Cancer Center, Collaborative Innovation Center for Cancer Personalized Medicine, Nanjing Medical University, Nanjing 211166 Jiangsu, China. ${ }^{15}$ Pulmonary and Critical Care Division, Massachusetts General Hospital, Department of Medicine, Harvard Medical School, Boston, MA 02114, USA. ${ }^{16}$ These authors contributed equally: Sipeng Shen, Yongyue Wei. ${ }^{17}$ These authors jointly supervised this work: Feng Chen, David C. Christiani. ${ }^{\circledR}$ email: zhangruyang@njmu.edu.cn; fengchen@njmu.edu.cn; dchris@hsph.harvard.edu
} 
a

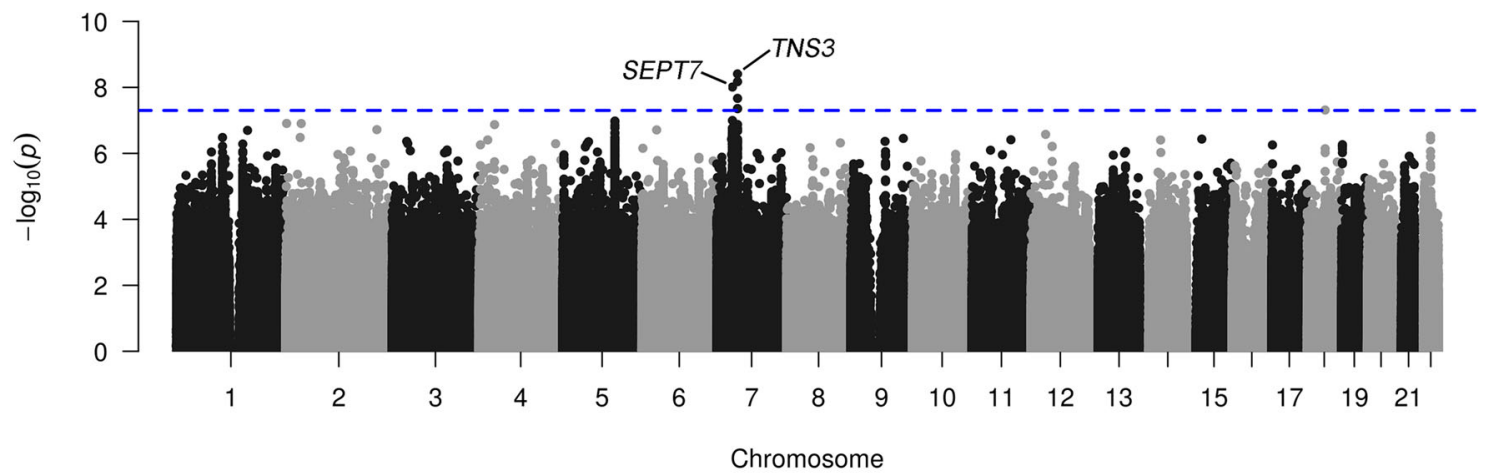

b

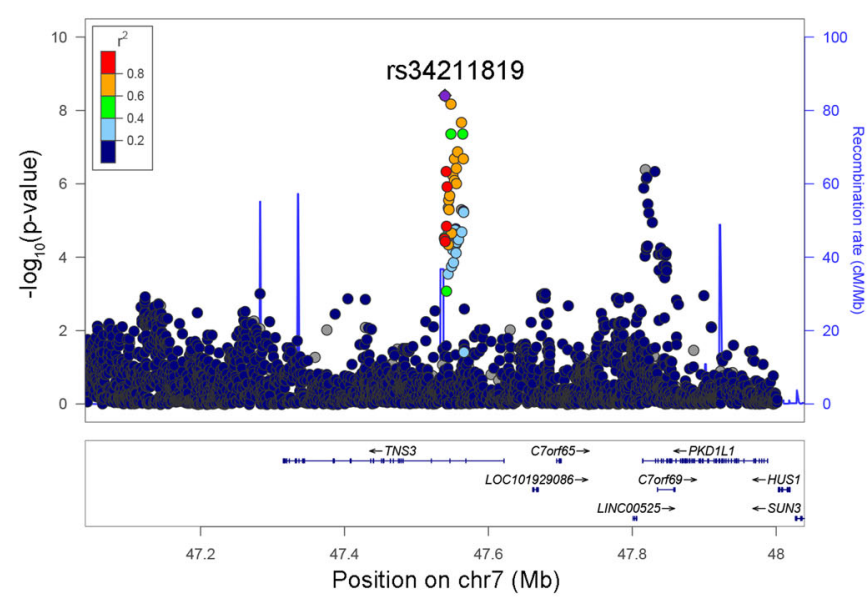

C

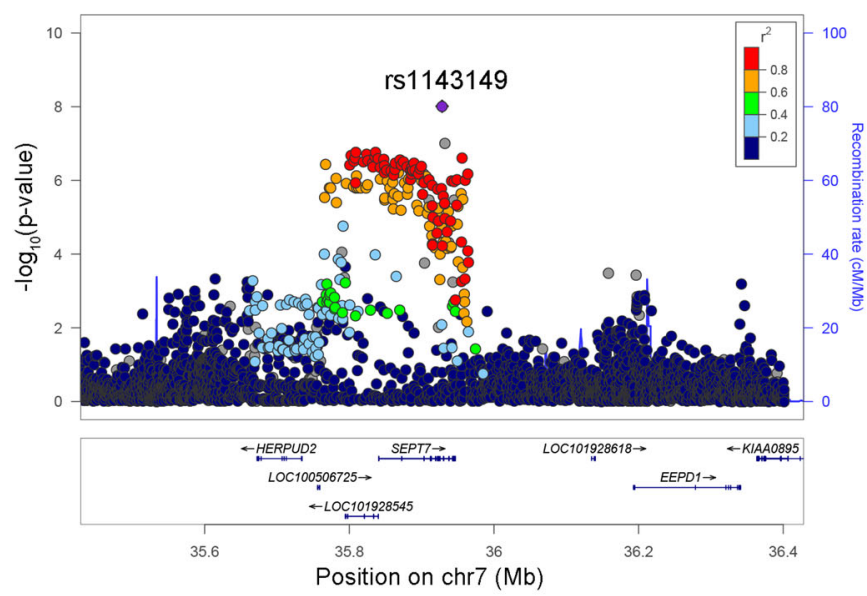

d

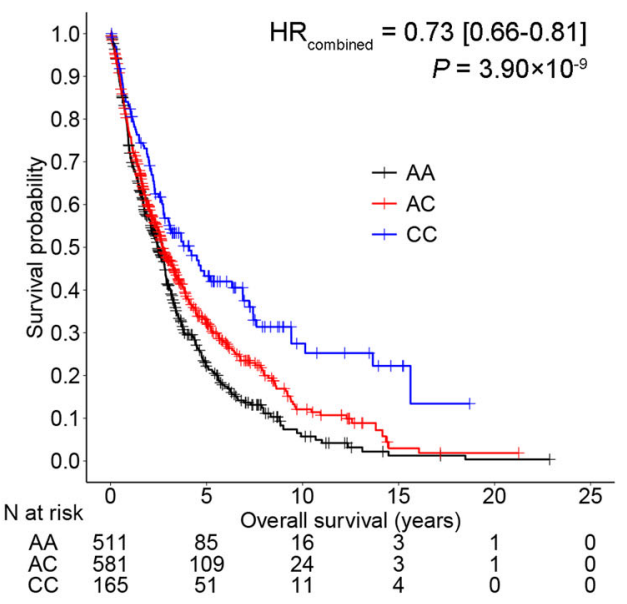

e

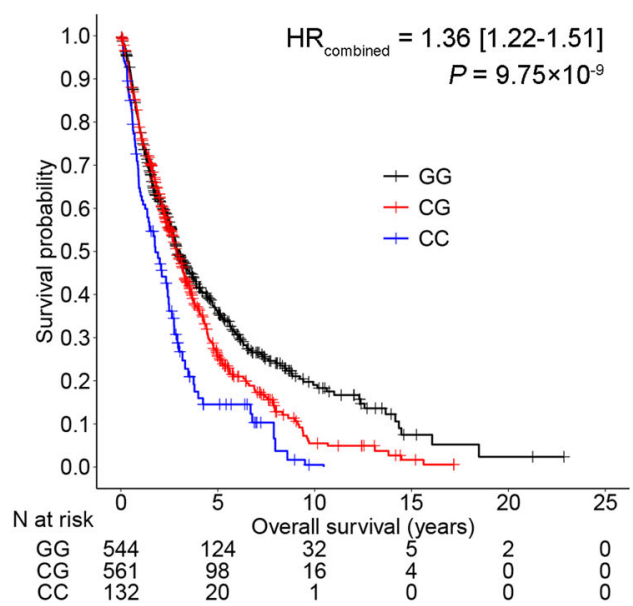

Fig. 1 Results of GWAS survival analysis. a Manhattan plot for survival analysis $P$ values. All genetic variants were coded using an additive model. SNPs with a minor allele frequency $>5 \%$, imputation $R^{2}>0.8$, and Hardy-Weinberg equilibrium $P>1 \times 10^{-5}$ were included. $\mathbf{b}$, $\mathbf{c}$ Regional association plots for rs34211819 in TNS3 and rs1143149 in SEPT7. The left-hand Y-axis shows - log10 transformation of the $P$-value of individual SNPs plotted against the chromosomal base-pair position with an expansion of $500 \mathrm{~kb}$ in the flanks of the SNP position. The right-hand $Y$-axis shows recombination rate estimated for European populations from HapMap Data Rel 22/phase II. d, e Kaplan-Meier survival curves for rs34211819 and rs1143149 in NSCLC patients by 0,1 , and 2 minor alleles. HRs, $95 \% \mathrm{Cl}$ and $P$ values were derived from Cox proportional hazards regression models adjusted for covariates.

rs34211819 was in an intron region of tensin-3 (TNS3), with an MAF of 0.35 ; rs1143149 was an intron variant of septin 7 (SEPT7), with an MAF of 0.34 . The regional plots (Fig. $1 \mathrm{~b}, \mathrm{c}$ ) present a cluster of significant prognostic SNPs that were moderately or highly correlated with rs34211819 and rs1143149 (Supplementary Table 1). Although the chromosome locations of the two SNPs were close, they had a very low correlation $\left(D^{\prime}=\right.$ $0.017, r^{2}=2.27 \times 10^{-5}$ ). The $C$ allele of $r s 34211819$ was 

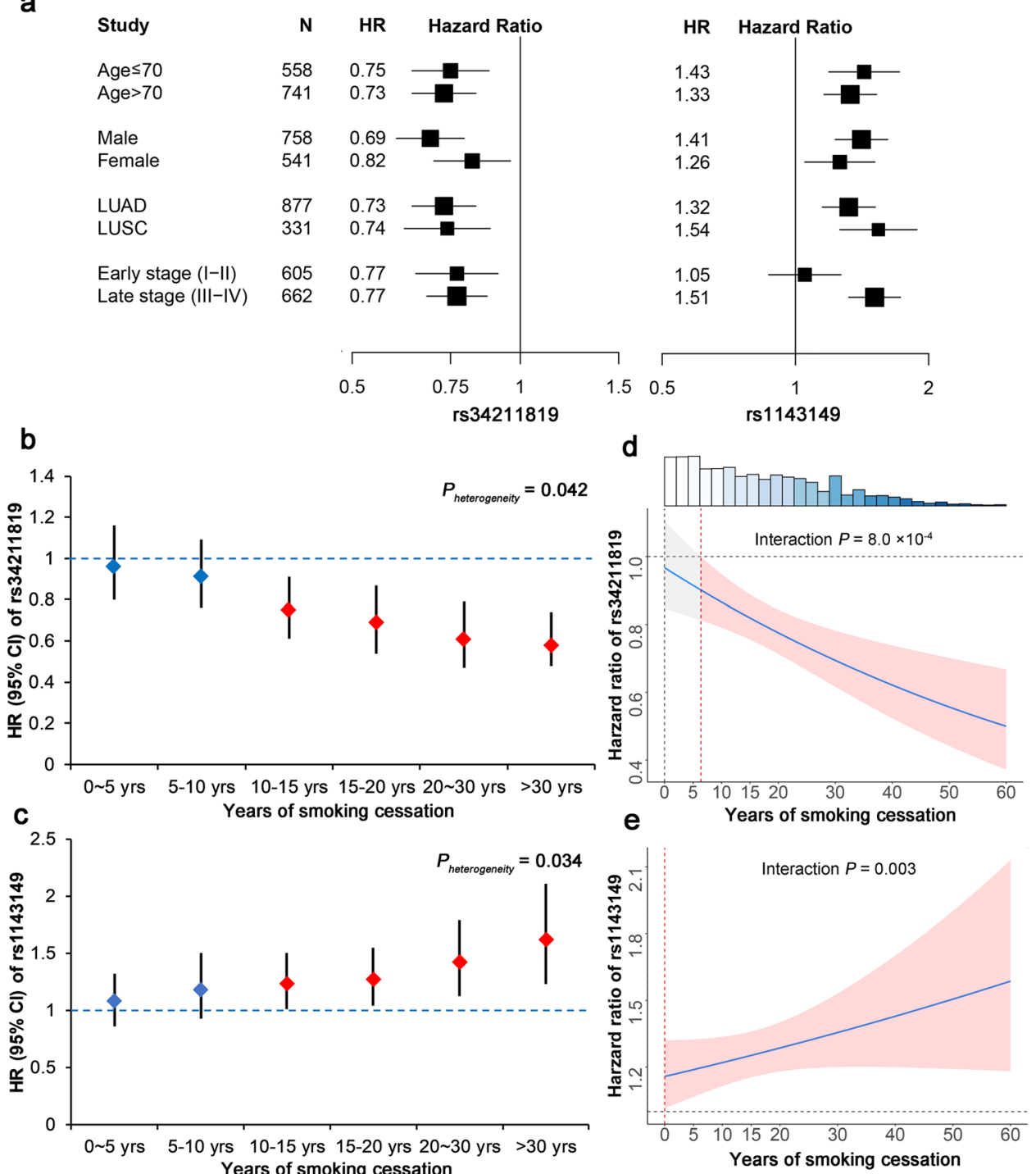

Fig. 2 Stratified analysis and interaction analysis with smoking cessation years. a Stratified analysis of rs34211819 (left) and rs1143149 (right) among different subgroups of long-term former smokers by age (divided by median value), gender, histology type, and clinical stage. b, c Stratified analysis of rs34211819 and rs1143149 by different years of smoking cessation in overall NSCLC patients. The left-hand Y-axis shows the effect size (HR and $95 \% \mathrm{Cl}$ ) derived from the Cox proportional hazards regression model. d, e Interaction plot for HRs of SNPs estimated based years of smoking cessation. The shaded area represents the $95 \% \mathrm{CI}$. Top histogram shows distribution of years of smoking cessation.

significantly associated with better survival $\left(\mathrm{HR}_{\text {discovery }}=0.66\right.$, $95 \% \mathrm{Cl}: 0.56-0.78, P=2.01 \times 10^{-7} ; \mathrm{HR}_{\text {validation }}=0.84,95 \% \mathrm{Cl}$ : $0.73-0.96, P=1.36 \times 10^{-2} ; H R_{\text {combined }}=0.73,95 \% \mathrm{Cl}: 0.66-0.81$, $P=3.90 \times 10^{-9}$ ) (Fig. 1d). In contrast, the C allele of rs 1143149 was associated with worse survival $\left(\mathrm{HR}_{\text {discovery }}=1.42,95 \% \mathrm{Cl}\right.$ : 1.16-1.60, $P=2.04 \times 10^{-6} ; \mathrm{HR}_{\text {validation }}=1.33,95 \% \mathrm{Cl}: 1.15-1.53$, $P=7.88 \times 10^{-5} ; H R_{\text {combined }}=1.36,95 \% \mathrm{Cl}: 1.22-1.51, P=9.75 \times$ $10^{-9}$ ) (Fig. 1e). The associations remained significant if SNPs were coded in co-dominant, dominant, and recessive models (Supplementary Table 2), indicating the robustness of our results toward coding. The same conclusion held with analyses of subgroups, defined by age, gender, histology, and clinical stage (Fig. 2a), as both SNPs were significantly associated with survival in almost all subgroups, except for rs1143149 among the early-stage patients.

\section{Genetic variants interaction with years of smoking cessation}

We performed a stratified analysis by years of smoking cessation for both long-term and short-term former smokers to evaluate the modifying effect of years of smoking cessation. As years of smoking cessation increased, the protective effect of rs34211819 and the detrimental effect of rs1143149 on survival were both elevated (Fig. 2b, c). These effects of two SNPs were only significant in long-term former smokers, and not in short-term former smokers, indicating significant heterogeneity between these two groups (rs34211819: $P_{\text {heterogeneity }}=0.042 ;$ rs1143149: $P_{\text {heterogeneity }}=$ 0.034). The trend test detected significant trends for both SNPs across different subgroups ( $r$ 34211819: $P_{\text {trend }}=0.003$; rs1143149: $P_{\text {trend }}=0.045$ ). Further, we detected significant interaction effects between SNPs and years of smoking cessation, a type of gene-environment interactions ( $r$ s4211819 $\times$ years: $P_{\text {interaction }}=$ $8.0 \times 10^{-4}$; rs $1143149 \times$ years: $\left.P_{\text {interaction }}=0.003\right)($ Fig. $2 \mathrm{~d}, \mathrm{e})$.

Evidence of association with lung cancer survival from multiomics studies

In meta-analysis of eQTL effects from GTEx and TCGA, the two SNPs had a significant cis-eQTL relationship with gene expression (TNS3: $\beta=-0.09,95 \% \mathrm{Cl}:-0.16$ to $-0.02, P=0.009$; SEPT7: $\beta=$ $0.04,95 \% \mathrm{Cl}: 0.01$ to $0.08, P=0.033$ ) (Fig. $3 \mathrm{~b}$ ). In gene expression 


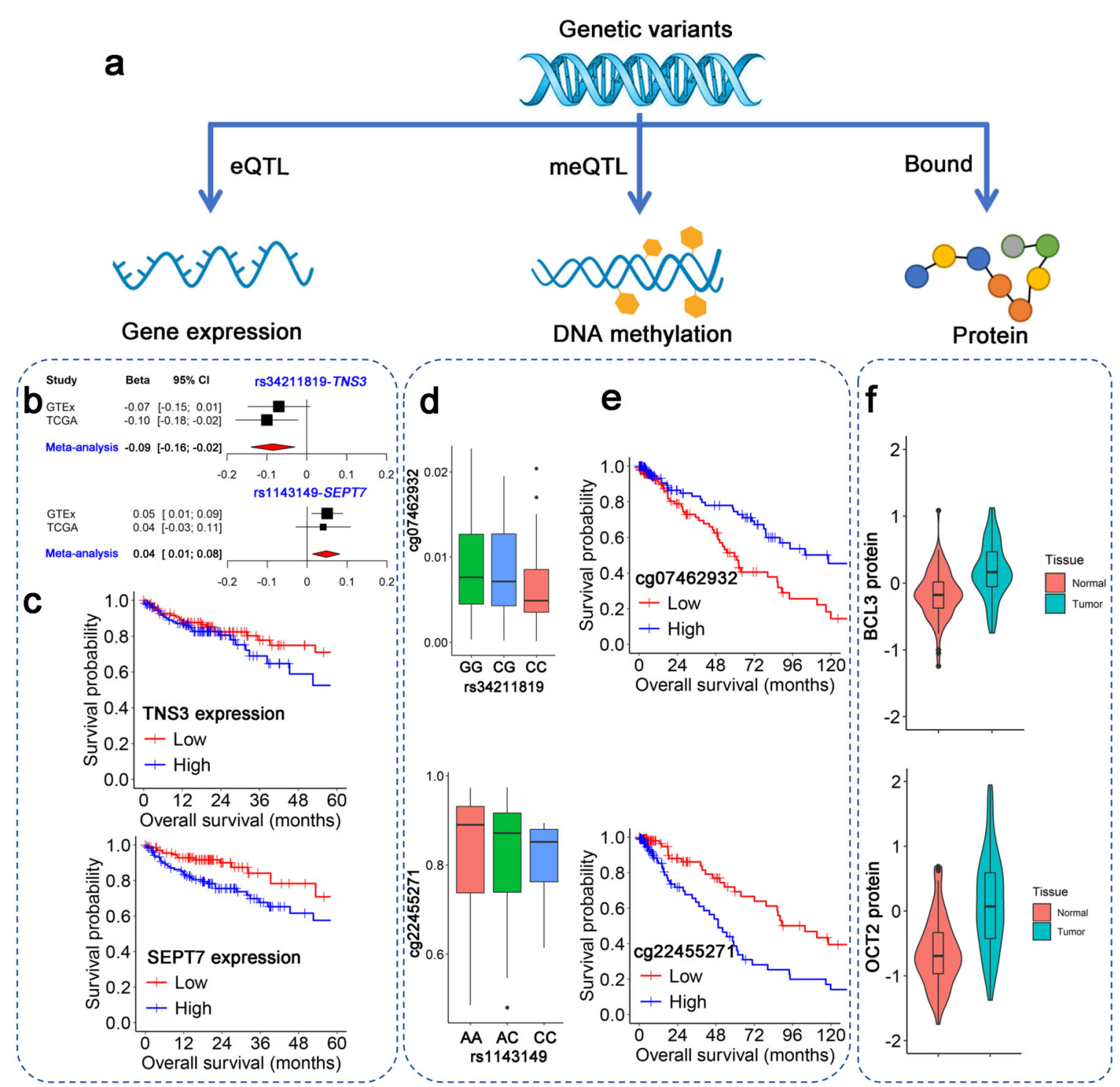

Fig. 3 Multi-omics analyses for TNS3 and SEPT7. a Multi-omics analyses flowchart. We observed significant eQTL/meQTL relationship, survival-related methylation, and expression patterns and upregulated proteins in NSCLC. b Significant eQTLs were observed for the two SNPs and cis genes. c Kaplan-Meier survival curves for expression of both genes using TCGA long-term former smoking patients. $\mathbf{d}$ meQTL boxplots of the two CpG probes and SNPs. e DNA methylation survival analysis for the two CpG probes. f Proteins BCL3 and OCT2 bound by SNPs also differed between tumor and normal tissues.

survival analysis of long-term former smokers, higher expression of TNS3 $(H R=1.84,95 \% \mathrm{Cl}: 1.01-3.34, P=0.045)$ and SEPT7 $(H R=$ $1.67,95 \% \mathrm{Cl}: 1.02-3.21, P=0.023)$ were significantly associated with worse survival in the TCGA database (Fig. 3c).

In DNA methylation analysis, we extracted $94 \mathrm{CpG}$ probes that were located within TNS3 and SEPT7. Two CpG sites, cg22455271 and cg07462932, had significant methylation QTL (meQTL) effects with $\mathrm{rs} 1143149(\beta=-0.08,95 \% \mathrm{Cl}:-0.14$ to $-0.01, P=0.009$, $q$-FDR $=0.034)$ and $\mathrm{rs} 34211819(\beta=-0.17,95 \% \mathrm{Cl}:-0.32$ to $-0.02, P=0.002, q$-FDR $=0.039$ ), respectively (Fig. $3 \mathrm{~d}$ ). They were significantly associated with survival in long-term former smokers $\left(\mathrm{HR}_{\mathrm{cg} 22455271}=2.40,95 \% \mathrm{Cl}: 1.47-3.93, P=2.5 \times 10^{-4}, q-\mathrm{FDR}=\right.$ $0.001 ; \quad \mathrm{HR}_{\mathrm{cg} 07462932}=0.47,95 \% \mathrm{Cl}: 0.30-0.74, \quad P=7.4 \times 10^{-4}$, $q$-FDR $=0.018$ ) (Fig. 3e and Supplementary Table 3).

We predicted the functional relevance by SNPinfo, RegulomeDB, and HaploReg v4.1 (Supplementary Table 4). rs34211819 in TNS3 had a high score of protein binding and could bind two proteins: B cell lymphoma 3 (BCL3) and octamer transcription factor 2 (OCT2).
In the CPTAC proteomics project, the two bound proteins BCL3 (fold change $=1.69, P=2.16 \times 10^{-5}$ ) and OCT2 (fold change $=2.21, P=$ $3.13 \times 10^{-13}$ ) were significantly upregulated in the lung cancer tumor tissues compared to the adjacent normal tissues (Fig. 3f).

\section{Genetic similarity across patients with different smoking statuses}

We first performed genetic similarity comparisons at the SNP level by extracting moderate-to-high signals from the results of survival analysis within different smoking subgroups. A total of 7789 independent SNPs was observed in the long-term former smokers, which were comparable to that of never-smokers $(n=7358)$ but $31.4 \%$ and $85.5 \%$ more than that of short-term former smokers $(n=$ $5343)$ and current smokers $(n=4198)$, respectively. No SNPs were shared across the four smoking subgroups. Long-term former smokers only had 23, 20, and 11 overlapping SNPs with never smokers, short-term former smokers, and current smokers, respectively (Fig. 4a), indicating that these significant prognostic SNPs for 

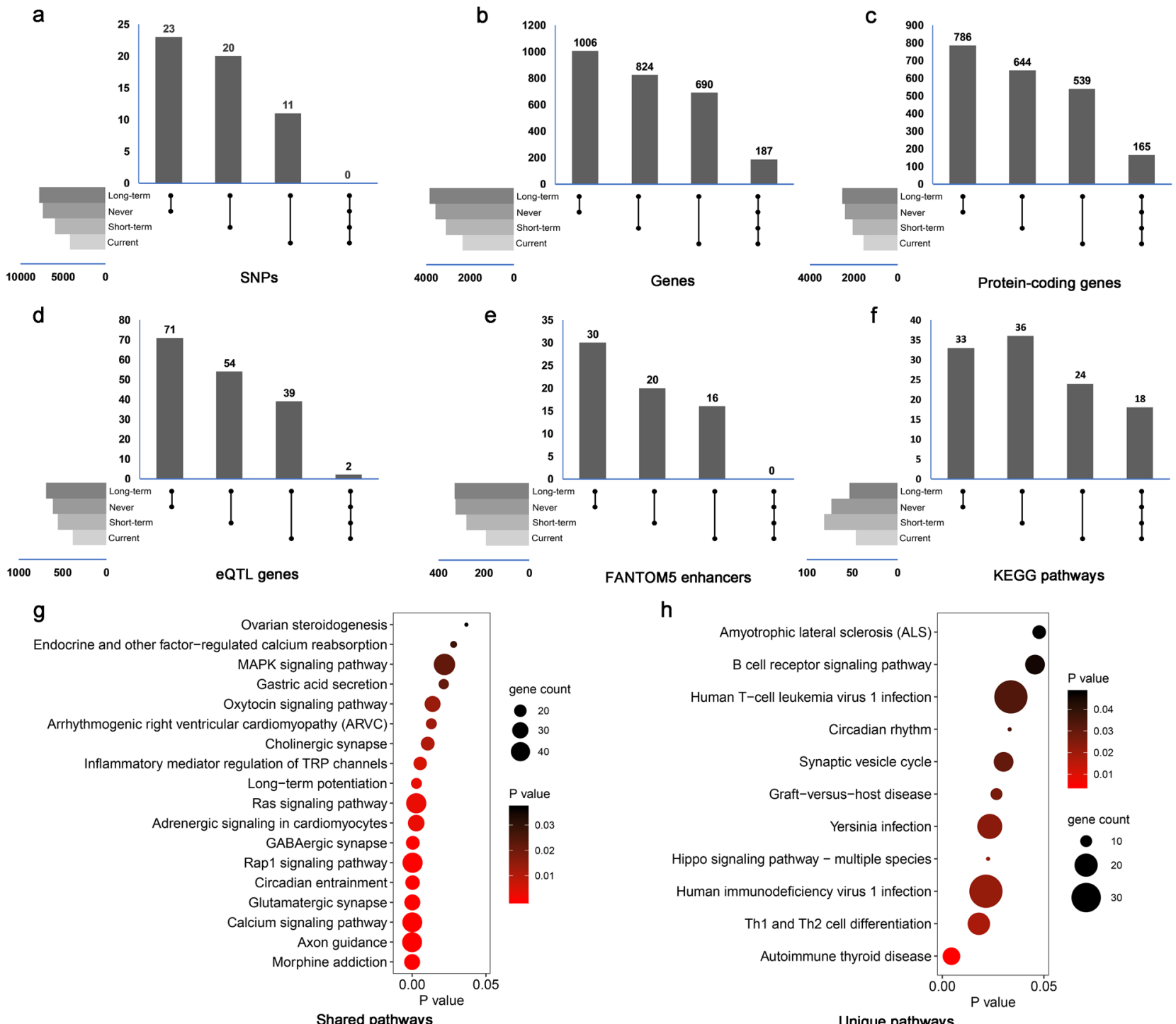

h

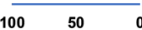

KEGG pathways

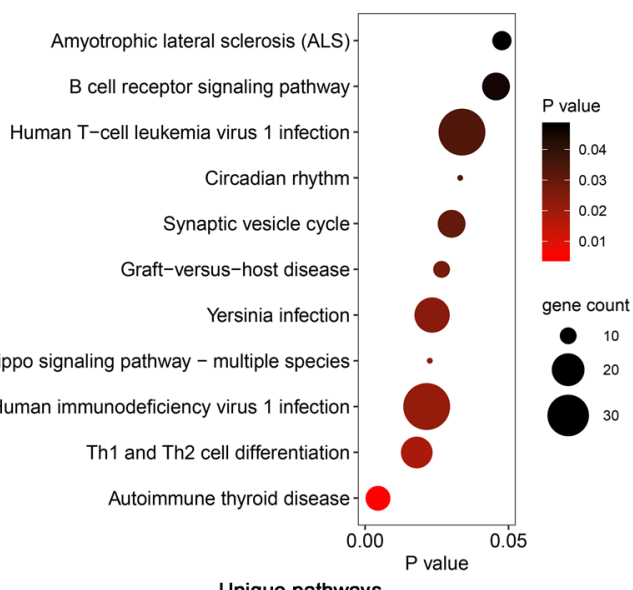

Fig. 4 Results of genetic similarity comparative analysis. Similarity comparison of SNPs with $P<10^{-3}$ at SNP level (a), germline-regulated gene level (b), germline-regulated protein-coding gene level (c), eQTL-related gene level (d), enhancer level (e), and KEGG pathway level (f), respectively. Protein-coding genes were defined by the GENCODE database. g-h Shared and unique KEGG pathways in long-term former smokers compared with never, short-term former, and current smokers.

NSCLC patients who were long-term former smokers seemed to differ from those for the other smoking groups.

For gene-level comparisons, these identified SNPs were assembled to genes within each subgroup (Fig. 4b). We identified 3,837 genes in long-term former smokers, and 1006 genes of them were shared with never-smokers, $22.1 \%$ more than those shared with short-term former smokers (824 genes) and $45.8 \%$ more than with current smokers ( 690 genes). A total of 187 genes were commonly shared by all the subgroups (Supplementary Table 5). Protein-coding genes showed these same patterns of similarity (Fig. 4c).

We further investigated the eQTL-related genes based on the GTEx lung tissue database (Fig. 4d) and enhancers from the FANTOM5 database (Fig. 4e). For long-term former smokers, the same trend was observed with germline-regulated genes, which may indicate the higher similarity with never-smoking subgroup than others. Only two eQTL-related genes were shared among all subgroups: ARHGAP15 and TSPAN9. We also performed sensitivity analysis under different thresholds $\left(P<10^{-4}\right.$ and $P<$ $10^{-5}$ ) and obtained the similar results (Supplementary Table 6).
Unique and shared pathways in long-term former smokers We explored Kyoto Encyclopedia of Genes and Genomes (KEGG) pathways in the gene set enrichment analysis of germlineregulated genes. A total of 53 pathways were significant in longterm former smokers (Fig. 4f). A total of 18 pathways were shared across all the subgroups, including the well-known signaling pathways such as mitogen-activated protein kinase, oxytocin, Ras, Rap1, and calcium pathways (Fig. 4g). However, 11 pathways were only significant in long-term former smokers, most of which were linked to immune function such as the B cell receptor signaling pathway, human $T$ cell leukemia virus 1 infection, human immunodeficiency virus 1 infection, and T helper type 1 and $T$ helper type 2 cell differentiation (Fig. 4h).

\section{DISCUSSION}

Understanding genetic risk factors in cancer is important for uncovering its underlying biological mechanisms ${ }^{11,12}$. Our genomewide investigation among long-term former smokers with NSCLC 
detected rs34211819 in TNS3 and rs1143149 in SEPT7 which were associated with survival. Significant interactions revealed that the effects of SNPs could be modified by years of smoking cessation, perhaps indicating opportunities for clinical adjuvant therapy with immunotherapies in patients with risk alleles, while an immune function relationship was found for cis-regulated genes or SNPbinding proteins. Multi-omics analyses provided evidence of their eQTL/meQTL relationships, survival-related methylation, and expression patterns and upregulated proteins in NSCLC. Furthermore, we observed higher similarities between long-term former smokers and never-smokers, compared to short-term former smokers or current smokers. The distinct SNP patterns in longterm former smokers were linked to immune signals.

Tobacco smoking is associated with worse outcomes in lung cancer, as it leads to downregulation of proinflammatory cytokines, immunosuppression, and anti-inflammatory effects mediated by oxidants, carbon monoxide, nicotine, and transcriptional modifying compounds, especially in lung tissues ${ }^{13,14}$. Chronic inhalation of cigarette smoke affects a wide range of immunological functions, including innate and adaptive immune responses ${ }^{15}$. As a result, the immune system may be suppressed as long as individuals are exposed to smoking regardless of years of quitting or smoking, as evidenced by the same signaling pathways shared by all the smoking subgroups in our study. Tobacco smoking can affect the immune system by chemically modifying signaling pathways as well as the extracellular matrix through acetylation, nitrosylation, carbonylation, and oxidation, thereby affecting cell survival, activation, and differentiation ${ }^{16}$.

The identified genes were associated with NSCLC survival at the genomic, epigenomic, and transcriptomic levels and were related to immunological functions. SEPT7 is a member of the septin family of GTP-binding proteins, which form higher-order filamentous structures and function primarily in spatial organization and compartmentalization of many cellular processes. SEPT7 is structurally related to RAS oncogenes, which promote tumorigenesis ${ }^{17}$. SEPT7 is also implicated in several types of cancer $^{18-20}$. While the septin family plays a critical role in cytokinesis ${ }^{21}$, SEPT7 is also involved in the cytoskeleton and participates in regulation of cytokinesis $^{22,23}$. Septin-deficient T cells fail to complete cytokinesis when prompted by pharmacological activation or cytokines. Meanwhile, SEPT7-deficient fibroblasts display incomplete cytokinesis and constitutive multinucleation by affecting the mitotic spindle and midbody rather than the contractile ring ${ }^{24}$. As a result, SEPT7 plays a crucial role in immune functions including cytokinesis and mitosis, which are closely related to molecular changes from smoking exposure.

Another locus at 7p12 is marked by an intronic SNP in TNS3, which encodes tensin-3, a member of a family of focal adhesionassociated proteins that regulate cell adhesion and migration ${ }^{25}$. This gene may be an activator of cell migration and a promoter of invasion in tumor metastasis ${ }^{26,27}$. Here, we found that TNS3 acts as an oncogene, affecting regulation of methylation in lung cancer. A TNS3 methylation pattern in the promoter region can silence expression in renal cell carcinoma ${ }^{28}$. Further, the binding proteins BCL3 on $19 q 13.32$ and OCT2 on $19 q 13.2$ of rs34211819 are strongly linked to immune function. $B C L 3$ translocates to the immunoglobulin alpha-locus in B cell chronic lymphocytic leukemia ${ }^{29}$. As an oncogene, it is an atypical member of the inhibitor of nuclear factor kappa B (NF-kB) family of proteins that can activate the NF-KB signaling cascade by directly binding to the transcription factors NFKB1 and NFKB2 ${ }^{30}$. It is unregulated by cytokines such as tumor necrosis factor alpha, interleukin 4 (IL-4), $\mathrm{IL}-1$, and IL- $6^{31}$. OCT2 acts as a DNA-binding transcriptional activator of immunoglobulin in B-lineage cells ${ }^{32}$. It enables B cells to respond normally to antigen receptor signals and mediate the physical interaction with $\mathrm{T}$ cells or to produce and respond to cytokines that are critical drivers of $B$ cell and $T$ cell differentiation during the immune response ${ }^{31,33}$.
Genetic similarity analysis of subgroups showed lower overlap at the SNP level but relatively higher overlap of germlineregulated genes. It is possible that each SNP is unique to each subgroup and acts as an eQTL to regulate cancer-specific genes. Additionally, the effects of somatic mutations driving lung cancer should be investigated. We also found higher similarities between long-term former smokers and never-smokers, which mainly included inflammation and immune mechanisms, as reported in the previous studies ${ }^{10,34}$.

This study had some limitations. First, although we used the largest lung cancer consortium to date, further external cohort validation with follow-up information and smoking cessation details is warranted. Second, we selected SNPs with moderate association strengths (from $P<10^{-5}$ to $P<10^{-3}$ ); although it was a reasonable approach ${ }^{35,36}$, some false-positive SNPs may have been included. More well-designed functional experiments are necessary to validate the biological functions.

This study also had several strengths. To the best of our knowledge, this is the first GWAS to investigate the effects of genetic variants on NSCLC patients among long-term former smokers. We included a large and relatively homogeneous study population with relatively complete and accurate follow-up, demographic, and clinical covariate information from ILCCO and HLCS. In addition, we investigated the association of candidate genes and lung cancer at the multi-omics levels, including genomics, transcriptomics, epigenomics, and proteomics. Similarity comparisons among different smoking subgroups revealed the shared genetics status at multiple levels.

In summary, our study demonstrated that TNS3 at 7p12.3 and SEPT7 at $7 \mathrm{p} 14.2$ are genetic regions associated with survival among long-term former smokers, and the findings with subgroup were related to immune function. Our results may shed light on the important roles of genetic architecture on cancer outcomes among long-term former smokers with NSCLC, a subpopulation that has been less studied.

\section{METHODS}

\section{Study population}

In accordance with the previous studies ${ }^{37-39}$, long-term former smokers were defined as patients who quitted smoking at least 10 years before diagnosis, whereas short-term former smokers quitted $<10$ years before diagnosis, and never smokers were those who smoked $<100$ cigarettes during their lifetime. To identify prognostic SNPs, we focused on long-term former smokers, whose characteristics are presented in Table 1. Patients in the discovery set were recruited from ILCCO, including the Cancer de Pulmon en Asturias study, Carotene and Retinol Efficacy Trial (CARET), Liverpool Lung Cancer Project, MD Anderson Cancer Center Study, and Mount-Sinai Hospital-Princess Margaret Study. While, patients in the independent validation set were recruited from HLCS (Supplementary Information). Approval for ILCCO studies was obtained from each of the participating institutional research ethics review boards. For HLCS, the Institutional Review Board of MGH and the Human Subjects Committee of the Massachusetts General Hospital and Harvard School of Public Health approved the study. All the participants were provided written informed consent to take part in the study.

Of the 6129 NSCLC patients with follow-up information totally, 4351 eligible cases were with available smoking information, including 504 never smokers, 1299 long-term former smokers, 687 short-term former smokers, and 1861 current smokers (Supplementary Table 7).

\section{OncoArray genotype quality control and imputation}

The ILCCO study and HLCS were originally designed and genotyped as case-control studies of lung cancer risk. In this study, we extracted all NSCLC patients from these two studies with survival information. Patient genotypes were generated using the Infinium OncoArray-500k BeadChip (Illumina, San Diego, CA, USA), with standard quality control procedures performed on all eligible individuals. Briefly, excluded were samples with $<95 \%$ completion and SNP assays with call rates $<95 \%$ or deviating from Hardy-Weinberg equilibrium $\left(P<10^{-6}\right)$. Only SNPs with minor allele 
frequencies (MAFs) $\geq 0.05$ mapping to autosomal chromosomes were included in the analysis. A total of 416,861 SNPs passed quality control ${ }^{40}$.

Genome-wide imputation following the Michigan Imputation Server pipeline ${ }^{41}$ was performed to estimate missing genotype information. We phased haplotypes with Eagle v2.3 using 1000 Genomes Project data (phase 3 ) as a reference panel ${ }^{42}$ and then performed imputations using the Minimac (version 3) software. SNPs with an imputation quality score $R^{2}<$ $0.8, \mathrm{MAF}<0.05$, or $P<10^{-6}$ for the Hardy-Weinberg equilibrium test were excluded from analyses.

Table 1. Demographic and clinical characteristics of long-term former smoking NSCLC patients.

\begin{tabular}{|c|c|c|c|}
\hline Characteristics & Discovery set & Validation set & Combined set \\
\hline Sample size & 566 & 733 & 1,299 \\
\hline Deaths (\%) & $387(68.4)$ & $500(68.2)$ & $887(68.2)$ \\
\hline $\begin{array}{l}\text { Median survival } \\
\text { years }(95 \% \mathrm{Cl})\end{array}$ & $2.14(1.71-2.51)$ & $2.87(2.69-3.31)$ & $2.64(2.38-2.86)$ \\
\hline Age (years) & $70.98 \pm 8.79$ & $70.01 \pm 9.22$ & $70.43 \pm 9.04$ \\
\hline Gender, male (\%) & $373(65.9)$ & $385(52.5)$ & $758(58.4)$ \\
\hline \multicolumn{4}{|l|}{ Histology (\%) } \\
\hline LUSC & $194(61.4)$ & $137(18.7)$ & $331(25.5)$ \\
\hline LUAD & $348(34.2)$ & $529(72.2)$ & $877(67.5)$ \\
\hline $\begin{array}{l}\text { NSCLC, not } \\
\text { specified }\end{array}$ & $24(4.2)$ & $67(9.1)$ & $91(7)$ \\
\hline \multicolumn{4}{|l|}{ Clinical stage (\%) } \\
\hline 1 & $207(36.5)$ & $258(35.2)$ & $465(35.8)$ \\
\hline II & $68(12.0)$ & $72(9.8)$ & $140(10.8)$ \\
\hline III & $131(23.1)$ & $165(22.5)$ & $296(22.8)$ \\
\hline IV & $138(24.3)$ & $228(31.1)$ & $366(28.2)$ \\
\hline $\begin{array}{l}\text { Pack-years of } \\
\text { smoking }\end{array}$ & $36.49 \pm 29.00$ & $37.49 \pm 29.03$ & $37.05 \pm 29.01$ \\
\hline $\begin{array}{l}\text { Years of smoking } \\
\text { cessation }\end{array}$ & $23.18 \pm 10.49$ & $23.66 \pm 10.39$ & $23.45 \pm 10.44$ \\
\hline
\end{tabular}

LUAD lung adenocarcinoma, LUSC lung squamous cell carcinoma, NSCLC non-small cell lung cancer.

\section{Two-stage GWAS survival analysis}

We used a two-stage strategy to identify significant prognostic SNPs for NSCLC patients who were long-term former smokers (Fig. 5). SNPs were encoded with an additive model (0: wild type; 1: heterozygosity; 2 homozygosity), unless otherwise stated. To quantify the association of each SNP with survival, we used the Cox proportional hazards regression model to evaluate its effect on survival, after adjusting for age, gender, clinical stage (I-IV), histology, pack-years of smoking, years of smoking cessation, and study center. Hazard ratio (HR) and $95 \%$ confidence interval (Cl) were described for mortality risk for patients per minor allele carried. To control for the confounding effects of population stratification, we also performed principal component analysis (PCA) and included the first three principal components in the model, although no population stratification was observed in the PCA plot (Supplementary Fig. 2). Following the same selection criteria commonly used in the previous GWAS survival studies of cancers ${ }^{43,44}$, we defined significant SNPs as those that met the following criteria: (i) $P \leq 10^{-5}$ in the discovery set; (ii) $P \leq 0.05$ in the validation set; and (iii) $P \leq 5 \times 10^{-8}$ in the combined set, reaching genome-wide significance. Kaplan-Meier curves were generated to illustrate survival differences between groups with different SNP genotypes, DNA methylation levels or gene expression levels.

\section{Gene-environment interaction analysis}

Since these SNPs significantly associated with prognosis of long-term former smokers with NSCLC, we further conducted gene-environment interaction analysis by estimating how their effects varied with increased years of smoking cessation. Specifically, we included the interaction terms between these SNPs and years of smoking cessation in the Cox proportional hazards regression model adjusted for the same covariates aforementioned.

\section{Multi-omics analysis}

We evaluated gene expression, DNA methylation of these significant SNPs, and further performed in silico function prediction and protein analysis.

To examine the eQTL relationships between SNPs and the expression levels of the corresponding genes, we evaluated their associations by using both the summary-level data from 383 lung tissue samples through the GTEx portal (V7 release) ${ }^{45}$ and the individual-level data from 208 NSCLC Caucasian patients who were long-term former smokers in The Cancer Genome Atlas (TCGA) database (Supplementary Table 8). The ciseQTL information in GTEx including normalized effect size, standard error, and nominal $p$ value for each SNP-expression pair was collected. Genotype data from TCGA were collected from the GDC Legacy Data Portal (Level 2, birdseed data), which were generated from Affymetrix Genome-Wide

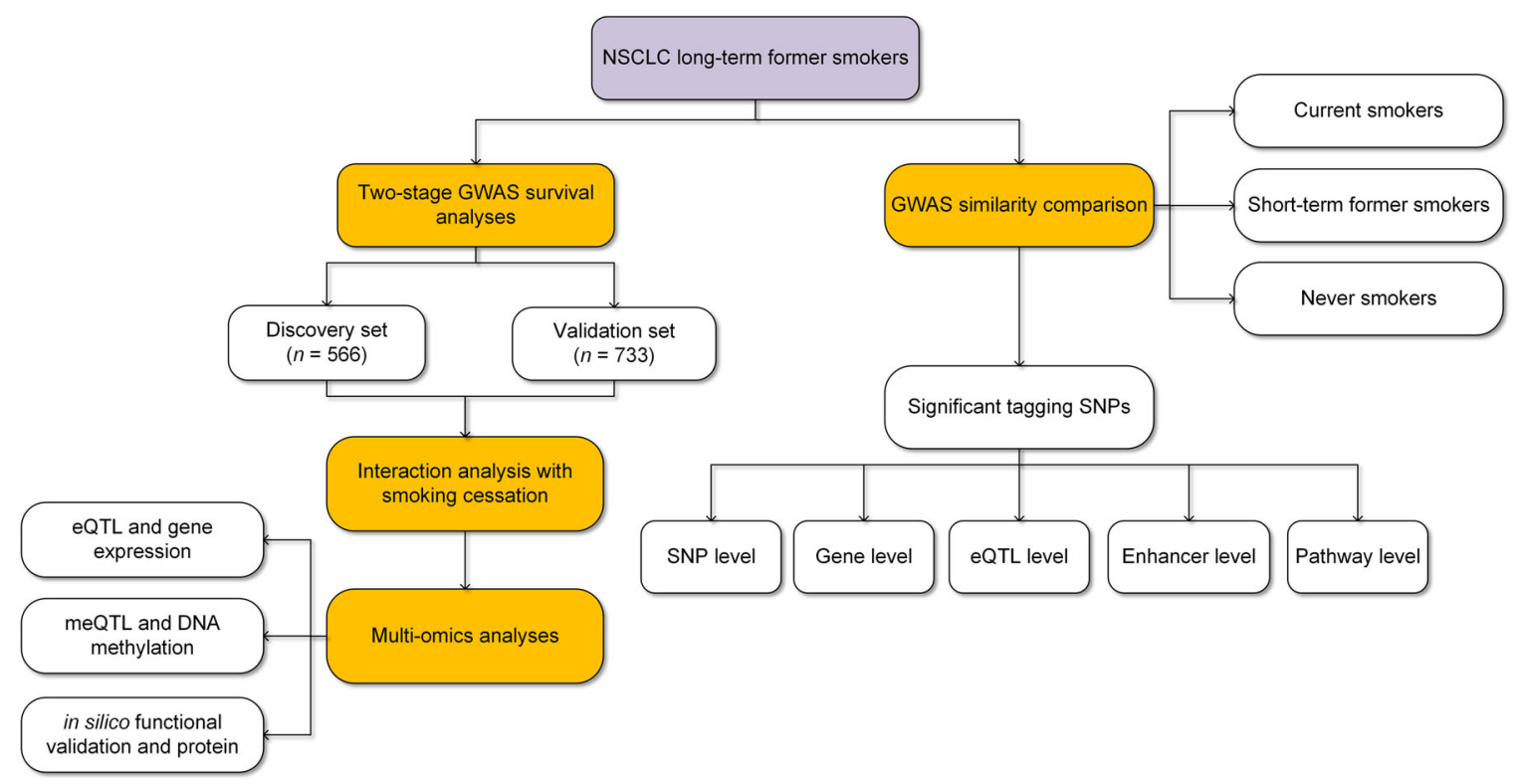

Fig. 5 Study workflow. Our study mainly included three parts: (1) GWAS survival study for long-term former smokers; (2) multi-omics study for candidate SNPs and genes; and (3) genetic similarity comparative analysis among different smoking status subgroups. 
Human SNP 6.0 Array. We performed the same quality control and imputation procedures as aforementioned. Gene expression values were normalized using the RNA-seq by expectation-maximization method ${ }^{46}$, and dichotomized, when needed, into low- and high-expression subgroups by the median values. To summarize the eQTL effects from TCGA and GTEx, we used meta-analysis with the fixed-effects model. The Cox proportional hazards regression model adjusted for the same covariates as aforementioned was utilized to evaluate the prognostic effects of gene expressions in tumor tissues in TCGA.

The association between SNP and DNA methylation was tested among 155 Caucasian patients who were long-term former smokers in TCGA. DNA methylation data were profiled using Illumina HumanMethylation450 BeadChips. The details of quality control were described $\mathrm{in}^{47}$. We used the linear regression model to assess meQTL effects and the Cox proportional hazards regression model to evaluate the association between methylation CpG probes (dichotomized by the median values) and survival. These models were adjusted for the same covariates. False discovery rate adjusted $P$ value ( $q$ value) was used to correct for multiple comparisons.

We used an in silico approach through SNPinfo ${ }^{48}$, RegulomeDB ${ }^{49}$, and HaploReg v4. $1^{50}$ to predict potential functions of the identified SNPs. We also compared protein levels among the 101 tumors paired with normal adjacent lung cancer tissue samples using Student's paired $t$ test in the Clinical Proteomic Tumor Analysis Consortium (CPTAC) project ${ }^{51}$. The data were normalized following the CPTAC Common Data Analysis Pipeline.

\section{Genetic similarity comparative analysis}

To assess genetic similarity of long-term former smoking patients with other smoking subgroups (e.g., never, short-term former, and current smokers), we performed GWAS survival analysis within each smoking subgroup using the Cox proportional hazards regression model adjusted for the same covariates aforementioned. We selected SNPs with moderate association strengths $\left(P<1 \times 10^{-3}\right)^{36}$. Genetic similarity comparisons were made at the SNP, gene, eQTL, enhancer, and pathway levels, respectively.

For gene-level analyses, germline-regulated genes were defined as the nearest genes using the Ensembl definitions on genome build GRCh37 (hg19), which annotated the genomic locations for each subgroup using gene start and stop coordinates ${ }^{52}$. All gene features were defined by GENCODE V $25^{53}$. We kept SNPs with no strong linkage disequilibrium (LD) using the PLINK indep-pairwise function; the $r^{2}$ used for all LD trimming was 0.5. eQTLs were collected from GTEx lung tissues as described above.

For enhancer-level analysis, the FANTOM5 human enhancer database was used to identify enhancer activities across most cell types and tissues ${ }^{54}$. SNPs located within a permissive enhancer region $\pm 1 \mathrm{~kb}$ were defined as the enhancer-related variants.

For pathway-level analysis, we performed gene enrichment pathway analysis based on the KEGG database. All enrichment analyses were performed using the R package clusterProfiler ${ }^{55}$.

All statistical analyses were performed using R (v3.5.2) or PLINK (v1.9).

\section{Reporting summary}

Further information on research design is available in the Nature Research Reporting Summary linked to this article.

\section{DATA AVAILABILITY}

The data generated and analyzed during this study are described in the following data record: https://doi.org/10.6084/m9.figshare.14229347 ${ }^{56}$.

This study utilized subsets of the Oncoarray Consortium-Lung Cancer Studies data available from the dpGap repository: https://identifiers.org/dbgap:phs001273.v3.p2 ${ }^{57}$. Specifically data from CAPUA study (CARET), the Roy Castle Lung Study (Liverpool Lung Cancer Project), the M.D. Anderson Cancer Center Study (MDACC study), the Mount-Sinai Hospital-Princess Margaret Study (MSH-PMH), and the Harvard Lung Cancer Study (HLCS) were accessed for this study. Prospective users of these data must apply for access, and details of how to apply can be found on the dataset landing page.

Functional prediction analyses of rs34211819 and rs1143149 (supporting Supplementary Table 4) are available from the HaploReg website https://pubs.broadinstitute.org/ mammals/haploreg/haploreg.php. De-identified participant demographic and phenotype data for lung adenocarcinoma patients are available from the National Cancer Institute GDC Legacy Archive https://portal.gdc.cancer.gov/legacy-archive/files/ 0f56f656-18f5-4648-97d9-bdbb0f2184a2. The CPTAC proteomics data file (CPTAC_Pro. $\mathrm{xlsx}$ ) can be openly accessed from the $\mathrm{NCl}$ Cancer Research Data Commons repository https://proteomic.datacommons.cancer.gov/pdc/study/PDC000153. The eQTL data
(GTEx_Analysis_v8_eQTL.tar) can be accessed directly from https://storage.googleapis. com/gtex_analysis_v8/single_tissue_qtl_data/GTEx_Analysis_v8_eQTL.tar.

\section{CODE AVAILABILITY}

The code that supports the findings of this study is available from the corresponding author on reasonable request.

Received: 9 September 2020; Accepted: 19 March 2021; Published online: 17 May 2021

\section{REFERENCES}

1. L., S. R., D., M. K. \& Ahmedin, J. Cancer statistics, 2017. CA: Cancer J. Clinicians 67, 7-30 (2017).

2. Boyle, P. \& Maisonneuve, P. Lung cancer and tobacco smoking. Lung Cancer 12, 167-181 (1995)

3. Murphy, S. E. et al. Tobacco biomarkers and genetic/epigenetic analysis to investigate ethnic/racial differences in lung cancer risk among smokers. NPJ Precis Oncol. 2, 17 (2018).

4. Moolgavkar, S. H. et al. Impact of reduced tobacco smoking on lung cancer mortality in the United States during 1975-2000. J. Natl Cancer Inst. 104, 541-548 (2012).

5. Sun, S., Schiller, J. H. \& Gazdar, A. F. Lung cancer in never smokers-a different disease. Nat. Rev. Cancer 7, 778 (2007).

6. Yano, T. et al. Never-smoking nonsmall cell lung cancer as a separate entity: clinicopathologic features and survival. Cancer 113, 1012-1018 (2008).

7. Couraud, S., Zalcman, G., Milleron, B., Morin, F. \& Souquet, P. J. Lung cancer in never smokers-a review. Eur. J. Cancer 48, 1299-1311, (2012).

8. Slatore, C. G., Au, D. H., Littman, A. J., Satia, J. A. \& White, E. Association of nonsteroidal anti-inflammatory drugs with lung cancer: results from a large cohort study. Cancer epidemiology, biomarkers \& prevention: a publication of the American Association for Cancer Research, cosponsored by the American Society of Preventive. Oncology 18, 1203-1207 (2009).

9. Huxley, R. et al. Impact of smoking and smoking cessation on lung cancer mortality in the Asia-Pacific region. Am. J. Epidemiol. 165, 1280-1286 (2007).

10. $\mathrm{Wu}, \mathrm{X}$. et al. Genome-wide association study of genetic predictors of overall survival for non-small cell lung cancer in never smokers. Cancer Res. 73, 4028-4038 (2013).

11. Shen, H., Zhu, M. \& Wang, C. Precision oncology of lung cancer: genetic and genomic differences in Chinese population. NPJ Precis Oncol. 3, 14 (2019).

12. Huang, X. et al. Genomic investigation of co-targeting tumor immune microenvironment and immune checkpoints in pan-cancer immunotherapy. NPJ Precis Oncol. 4, 29 (2020).

13. Chen, H., Cowan, M. J., Hasday, J. D., Vogel, S. N. \& Medvedev, A. E. Tobacco smoking inhibits expression of proinflammatory cytokines and activation of IL$1 \mathrm{R}$-associated kinase, p38, and NF-kappaB in alveolar macrophages stimulated with TLR2 and TLR4 agonists. J. Immunol. 179, 6097-6106 (2007).

14. Smith, C. J. \& Hansch, C. The relative toxicity of compounds in mainstream cigarette smoke condensate. Food Chem. Toxicol. 38, 637-646 (2000).

15. Sopori, M. Effects of cigarette smoke on the immune system. Nat. Rev. Immunol. 2, 372-377 (2002).

16. Stampfli, M. R. \& Anderson, G. P. How cigarette smoke skews immune responses to promote infection, lung disease and cancer. Nat. Rev. Immunol. 9, 377-384 (2009).

17. Angelis, D. \& Spiliotis, E. T. Septin mutations in human cancers. Front Cell Dev. Biol. 4, 122 (2016).

18. Connolly, D., Abdesselam, I., Verdier-Pinard, P. \& Montagna, C. Septin roles in tumorigenesis. Biol. Chem. 392, 725-738 (2011).

19. Russell, S. \& Hall, P. Do septins have a role in cancer? Br. J. Cancer 93, 499 (2005).

20. Liu, M., Shen, S., Chen, F., Yu, W. \& Yu, L. Linking the septin expression with carcinogenesis. Mol. Biol. Rep. 37, 3601-3608 (2010).

21. Wang, X. et al. The role of septin 7 in physiology and pathological disease: a systematic review of current status. J. Cell Mol. Med 22, 3298-3307 (2018).

22. Hou, M., Liu, X., Cao, J. \& Chen, B. SEPT7 overexpression inhibits glioma cell migration by targeting the actin cytoskeleton pathway. Oncol. Rep. 35, 2003-2010 (2016).

23. Abbey, M. et al. GTPase domain-driven dimerization of SEPT7 is dispensable for the critical role of septins in fibroblast cytokinesis. Sci. Rep. 6, 20007 (2016).

24. Menon, M. B. et al. Genetic deletion of SEPT7 reveals a cell type-specific role of septins in microtubule destabilization for the completion of cytokinesis. PLoS Genet. 10, e1004558 (2014).

25. Touaitahuata, H. et al. Tensin 3 is a new partner of Dock5 that controls osteoclast podosome organization and activity. J. Cell Sci. 129, 3449-3461 (2016). 
26. Qian, X. et al. The Tensin-3 protein, including its $\mathrm{SH} 2$ domain, is phosphorylated by Src and contributes to tumorigenesis and metastasis. Cancer Cell 16, 246-258 (2009).

27. Cao, X. et al. A phosphorylation switch controls the spatiotemporal activation of Rho GTPases in directional cell migration. Nat. Commun. 6, 7721 (2015).

28. Carter, J. A., Gorecki, D. C., Mein, C. A., Ljungberg, B. \& Hafizi, S. CpG dinucleotidespecific hypermethylation of the TNS3 gene promoter in human renal cell carcinoma. Epigenetics 8, 739-747 (2013).

29. Ohno, H., Takimoto, G. \& McKeithan, T. W. The candidate proto-oncogene bcl-3 is related to genes implicated in cell lineage determination and cell cycle control. Cell 60, 991-997 (1990).

30. Wulczyn, F. G., Naumann, M. \& Scheidereit, C. Candidate proto-oncogene bcl-3 encodes a subunit-specific inhibitor of transcription factor NF-KB. Nature 358, 597 (1992).

31. Maldonado, V. \& Melendez-Zajgla, J. Role of Bcl-3 in solid tumors. Mol. Cancer 10 152 (2011)

32. Staudt, L. M. et al. Cloning of a lymphoid-specific cDNA encoding a protein binding the regulatory octamer DNA motif. Science 241, 577-580 (1988).

33. Corcoran, L. et al. Oct 2 and Obf1 as facilitators of B:T cell collaboration during a humoral immune response. Front. Immunol. 5, 108 (2014).

34. Pu, X. et al. Predictors of survival in never-smokers with non-small cell lung cancer: a large-scale, two-phase genetic study. Clin. Cancer Res. 18, 5983-5991 (2012).

35. Jia, P., Wang, L., Meltzer, H. Y. \& Zhao, Z. Pathway-based analysis of GWAS datasets: effective but caution required. Int. J. Neuropsychopharmacol. 14, 567-572 (2011)

36. O'Brien, T. D., Jia, P., Caporaso, N. E., Landi, M. T. \& Zhao, Z. Weak sharing of genetic association signals in three lung cancer subtypes: evidence at the SNP, gene, regulation, and pathway levels. Genome Med. 10, 16 (2018).

37. Ellickson, P. L., Mcguigan, K. A. \& Klein, D. J. Predictors of late-onset smoking and cessation over 10 years. J. Adolesc. Health 29, 101-108 (2001).

38. Edwards, R. The problem of tobacco smoking. BMJ 328, 217-219 (2004).

39. Cornuz, J., Feskanich, D., Willett, W. C. \& Colditz, G. A. Smoking, smoking cessation, and risk of hip fracture in women. Am. J. Med. 106, 311-314 (1999).

40. McKay, J. D. et al. Large-scale association analysis identifies new lung cancer susceptibility loci and heterogeneity in genetic susceptibility across histological subtypes. Nat. Genet. 49, 1126 (2017).

41. Das, S. et al. Next-generation genotype imputation service and methods. Nat. Genet. 48, 1284-1287 (2016).

42. Loh, P.-R. et al. Reference-based phasing using the Haplotype Reference Consortium panel. Nat. Genet. 48, 1443 (2016).

43. $\mathrm{Wu}, \mathrm{C}$. et al. Genome-wide association study of survival in patients with pancreatic adenocarcinoma. Gut 63, 152-160 (2014).

44. Tang, H. et al. Genetic polymorphisms associated with pancreatic cancer survival: a genome-wide association study. Int. J. Cancer 141, 678-686 (2017).

45. Lonsdale, J. et al. The genotype-tissue expression (GTEx) project. Nat. Genet. 45 , 580 (2013)

46. Li, B. \& Dewey, C. N. RSEM: accurate transcript quantification from RNA-Seq data with or without a reference genome. BMC Bioinform. 12, 323 (2011).

47. Shen, S. et al. A multi-omic study reveals BTG2 as a reliable prognostic marker for early-stage non-small cell lung cancer. Mol. Oncol. 12, 913-924 (2018).

48. Xu, Z. \& Taylor, J. A. SNPinfo: integrating GWAS and candidate gene information into functional SNP selection for genetic association studies. Nucleic Acids Res. 37, W600-W605 (2009)

49. Boyle, A. P. et al. Annotation of functional variation in personal genomes using Regulome DB. Genome Res. 22, 1790-1797 (2012).

50. Ward, L. D. \& Kellis, M. HaploReg: a resource for exploring chromatin states, conservation, and regulatory motif alterations within sets of genetically linked variants. Nucleic Acids Res. 40, D930-D934 (2011).

51. Gillette, M. A. et al. Proteogenomic characterization reveals therapeutic vulnerabilities in lung adenocarcinoma. Cell 182, 200-225 e235 (2020).

52. Zerbino, D. R., Wilder, S. P., Johnson, N., Juettemann, T. \& Flicek, P. R. The ensemble regulatory build. Genome Biol. 16, 56 (2015).

53. Frankish, A. et al. GENCODE reference annotation for the human and mouse genomes. Nucleic Acids Res. 47, D766-d773 (2019).

54. Andersson, R. et al. An atlas of active enhancers across human cell types and tissues. Nature 507, 455 (2014).

55. Yu, G., Wang, L.-G., Han, Y. \& He, Q.-Y. clusterProfiler: an R package for comparing biological themes among gene clusters. Omics: J. Integr. Biol. 16, 284-287 (2012).
56. Shen, S. et al. Metadata record for the article: a multi-omics study links TNS3 and SEPT7 to long-term former smoking NSCLC survival. figshare https://doi.org/ 10.6084/m6089.figshare.14229347 (2021).

57. Oncoarray Consortium-Lung Cancer Studies. dbGaP https://identifiers.org/ dbgap:phs001273.v3.p2.

\section{ACKNOWLEDGEMENTS}

This study was funded by the National Key Research and Development Program of China (2016YFE0204900 to F.C.), National Natural Science Foundation of China (81530088 to F.C. and 81973142 to Y.W.), Jiangsu Planned Projects for Postdoctoral Research Funds (2020Z019 to S.S.), Natural Science Foundation of Jiangsu Province (BK20191354 to R.Z.), Natural Science Foundation of the Jiangsu Higher Education Institutions of China (18KJB310011 to R.Z.), China Postdoctoral Science Foundation (2020M681671 to S.S. and 2018M633767 to R.Z.), US National Institutes of Health (CA209414 to D.C.C. and CA203654 to C.I.A. and R.J.H.), and Priority Academic Program Development of Jiangsu Higher Education Institutions (PAPD). CARET is funded by the National Cancer Institute, National Institutes of Health through grants U01-CA063673, UM1-CA167462, and U01-CA167462. R.Z. was partially supported by the Outstanding Young Teachers Training Program of Nanjing Medical University.

\section{AUTHOR CONTRIBUTIONS}

S.S., Y.W., D.C.C., F.C., and R.Z. contributed to the study design. A.T., C.C., J.F., R.H., G.L., D.Z., C.A., T.F., L.S., and D.C.C. contributed to the data collection. S.S., R.Z., Y.W., W.D., X.D., L.L., D.Y., and Y.Z. performed the statistical analysis and interpretation. S.S. and R.Z. drafted the manuscript. S.S., R.Z., Y.W., R.H., Z.H., H.S., and Y.L. revised the manuscript. All authors contributed to critical revision of the final manuscript and approved the final version of the manuscript.

\section{COMPETING INTERESTS}

The authors declare no competing interests.

\section{ADDITIONAL INFORMATION}

Supplementary information The online version contains supplementary material available at https://doi.org/10.1038/s41698-021-00182-3.

Correspondence and requests for materials should be addressed to R.Z., F.C. or D. C.C.

Reprints and permission information is available at http://www.nature.com/ reprints

Publisher's note Springer Nature remains neutral with regard to jurisdictional claims in published maps and institutional affiliations.

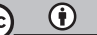

Open Access This article is licensed under a Creative Commons Attribution 4.0 International License, which permits use, sharing, adaptation, distribution and reproduction in any medium or format, as long as you give appropriate credit to the original author(s) and the source, provide a link to the Creative Commons license, and indicate if changes were made. The images or other third party material in this article are included in the article's Creative Commons license, unless indicated otherwise in a credit line to the material. If material is not included in the article's Creative Commons license and your intended use is not permitted by statutory regulation or exceeds the permitted use, you will need to obtain permission directly from the copyright holder. To view a copy of this license, visit http://creativecommons. org/licenses/by/4.0/.

(c) The Author(s) 2021 